\title{
Resistência ao movimento e atividade eletromiográfica dos músculos flexores e extensores de cotovelo em pacientes hemiparéticos espásticos submetidos à crioterapia e estimulação elétrica neuromuscular
}

Débora Daisy da Silva*, Ana Carolina Lacerda Borges, Mário Oliveira Lima, Fernanda Pupio Silva Lima, Sérgio Takeshi Tatsukawa de Freitas, Daniel Vilela Nogueira, Paulo Roberto Garcia Lucareli, Alderico Rodrigues de Paula Junior, José Carlos Cogo

Resumo A espasticidade quase sempre coexiste a um Acidente Vascular Encefálico (AVE), devido à lesão do motoneurônio superior, provocando um impacto nas atividades da vida diária e na qualidade de vida dos pacientes. Por esse motivo é de suma importância o controle do tônus muscular, que pode ser alcançado por meio de recursos fisioterapêuticos, como a crioterapia e a Estimulação Elétrica Neuromuscular (EENM). Como demonstrado em diversas pesquisas na área, esses recursos são vantajosos pelos efeitos fisiológicos que proporcionam, porém, são insuficientes os trabalhos científicos que forneçam embasamento para o tratamento da espasticidade dos membros superiores e, evidenciem o tempo de duração dos efeitos fisiológicos após aplicação desses recursos. Nesse sentido, o presente trabalho vem contribuir para o estabelecimento de protocolo de aplicação de crioterapia e EENM em indivíduos portadores de hemiparesia espástica e, informar a duração dos efeitos após submissão às terapias, por meio da análise da resistência ao movimento dos músculos flexores e extensores de cotovelo antes, depois, 10, 20 e 30 minutos após a aplicação de crioterapia e EENM. Participaram do estudo 15 pacientes com diagnóstico de AVE e diagnóstico fisioterapêutico de hemiparesia espástica, de ambos os sexos, com idade média de $56 \pm 16$ anos. A avaliação foi realizada antes e após a terapia, e nos instantes de 10, 20 e 30 minutos após o término de sua aplicação, e consistiu na captação da atividade eletromiográfica por meio da Eletromiografia (EMG) de superfície e da resistência ao movimento por meio do Dinamômetro Isocinético, no modo passivo nas velocidades angulares de $30 \%$ e $150 \%$ s. Embora não estatisticamente significativos $(p<0,05)$ os resultados evidenciam uma redução da resistência à movimentação passiva dos flexores e extensores de cotovelo após a aplicação de crioterapia e EENM, comprovando a eficácia dos recursos fisioterapêuticos na redução da resistência à movimentação passiva de indivíduos hemiparéticos espásticos, embora, apresentem efeitos diferentes com relação ao tempo.

Palavras-chave Acidente vascular encefálico, Espasticidade, Dinamômetro isocinético, Eletromiografia, Crioterapia, Estimulação elétrica neuromuscular (EENM).

\section{Resistance to movement and electromyographic activity of the elbow flexor and extensor muscles in spastic hemiparetic patients following cryotherapy and electrical stimulation}

\footnotetext{
Abstract Spasticity often coexists with stroke due to an upper motor neuron lesion, which impacts the patient's quality of life. Therefore, the control of muscle tone through physical therapy such as cryotherapy and Neuromuscular Electrical Stimulation (NMES) is extremely important. Several studies have demonstrated their advantageous physiological effects. However, these studies have not provided sufficient scientific proof for the treatment of upper limb spasticity and the duration of the physiological effects after the therapy. Therefore, this work helps to establish an application protocol for cryotherapy and NMES in individuals with spastic hemiparesis. The resistance to movement of the elbow flexor and extensor muscles before and after application of cryotherapy and NMES was used to determine the effect of the therapies. The study included 15 stroke patients that required physical therapy for spastic hemiparesis. They included both sexes, mean age of $56 \pm 16$. An evaluation was performed before and after therapy, as well as 10, 20, and 30 minutes following the application, by means of surface electromyography (EMG). The resistance to movement was measured with an Isokinetic Dynamometer in the passive mode at an angular velocity of 30 and $150 \%$ s. Although not statistically significant $(p<0.05)$, the results showed a reduced resistance to passive movement of the elbow flexors and extensors after cryotherapy and NMES, allowing us to conclude that physical therapy effectively reduces the resistance to passive movement in spastic hemiparetic patients, however, they have different effects with respect to time.
}

Keywords Stroke, Spasticity, Isokinetic dynamometer, Electromyography, Cryotherapy, Neuromuscular electrical stimulation (NMES).

*e-mail: deboradaisy@hotmail.com

Recebido: 31/01/2012 / Aceito: 20/04/2012 


\section{Extended Abstract}

\section{Introduction}

Responsible for a large proportion of disability and death, stroke is considered a serious public health problem. Stroke refers to the interruption of cerebral blood flow, resulting in neurological symptoms in the artery and affected region. An upper motor neuron lesion, which almost always coexists with stroke spasticity, can be expressed by increased resistance to passive stretching and decreased recruitment of motor units. Several physical therapy resources can be used to facilitate the control of muscle tone, including cryotherapy, involving the transfer of thermal energy through tissues, and Neuromuscular Electrical Stimulation (NMES), which is the application of electricity into the human body for therapeutic purposes. This study assessed the resistance to movement and electromyographic activity of the flexor and extensor muscles of spastic hemiparetic elbow, which was subjected to cryotherapy and NMES.

\section{Material and Methods}

This randomized, controlled, cross-over clinical trial was conducted at the Biodynamics Laboratory at the Universidade do Vale do Paraiba (UNIVAP). The study included 15 stroke patients who required physical therapy for spastic hemiparesis. Patients of both sexes, with mean age of $56 \pm 16$ were treated with cryotherapy and electrical stimulation. The evaluation consisted of the simultaneous capture of electromyographic activity and resistance to passive movement. The patients were evaluated before and after therapy, as well as 10,20, and 30 minutes after the application of NMES and cryotherapy. The electromyographic signal was captured with an EMG410C electromyograph (EMG System do Brasil Ltda). The resistance to movement of the elbow flexor and extensor muscles was obtained by a Computerized Isokinetic Dynamometer (Biodex System ${ }^{\circledR} 3$ ), in passive mode and with angular velocities of 30 and $150 \%$ s. To check for statistical differences, analysis of variance (one-way ANOVA) and t-test with a significance level of $p<0.05$ was used. The percentage of torque and RMS were measured to check the response of spastic individuals when subjected to the two therapies.

\section{Results}

Although not statistically significant $(p<0.05)$, the results showed a reduced resistance to passive movement of the elbow flexors and extensors after cryotherapy and electrical stimulation. Cryotherapy was overall more effective with the maximum spasticity reduction of the biceps muscle at 10 minutes after completion of therapy (59.8\%). NMES showed better results 20 and 30 minutes after completion of therapy (30.4 and $24.1 \%$, respectively). This indicates an acute effect of cryotherapy and a delayed effect of NMES. In addition to the spasticity reduction, the relation between the agonist and antagonist improved following cryotherapy, as evidenced by increased electromyographic activity of the triceps (21.9\%) 30 minutes after the completion of therapy.

\section{Discussion}

Spasticity is one of the functional disorders resulting from stroke. The upper limbs are the most affected and their rate of functional recovery is lower when compared with independent gait training. Cryotherapy and NMES provide satisfactory results in reducing spasticity. Our results showed decreased resistance to passive movement and increased RMS values, which confirms data presented by other authors. The efficacy of NMES in reducing spasticity has been observed in previous studies and is possibly due to a reduction in activity through the recurrent inhibition of alpha motoneuron. The effects of cryotherapy can be attributed to the reduction of synaptic transmission due to decreased velocity of nerve conduction. The uniqueness of this research was to demonstrate the physiological effect up to 30 minutes after the end of the proposed therapies.

\section{Conclusion}

The results indicated that the therapeutic resources promoted reduced resistance to passive movement of the elbow flexors and extensors; however, they have different effects with respect to time. Thus, the cryotherapy and NMES protocols in this study can be used as supporting methods for treating spasticity. 


\section{Introdução}

O Acidente Vascular Encefálico (AVE) é uma condição neurológica súbita decorrente de uma isquemia cerebral localizada ou hemorragia (World..., 2006). A deficiência motora, que afeta cerca de 80\% dos pacientes com AVE (Kumar et al., 2006; Sommerfeld et al., 2004), é proveniente de lesões do trato piramidal associadas a lesões do sistema extrapiramidal. É descrita como síndrome do neurônio motor superior (Sommerfeld et al., 2004; Urban et al., 2010), caracterizada clinicamente pela presença de espasticidade, sinal de Babinski, reflexos cutâneos e autonômicos exagerados, espasmos involuntários de flexores e extensores, clônus, posturas anormais e paresia. Essas alterações podem desencadear distúrbios do movimento, causar dor e contraturas e limitações funcionais (Kumar et al., 2006; Nuyens et al., 2002).

Alguns autores sugerem que a espasticidade é uma desordem motora caracterizada por hiperexcitabilidade dos reflexos de estiramento, exacerbação dos reflexos profundos e aumento do tônus muscular, tendo como característica o aumento da resistência ao estiramento passivo dependente de velocidade (Alibiglou et al., 2008; Bensmail et al., 2010; Dietz e Sinkjaer, 2007; Lindberg et al., 2009). Presente em 19-39\% dos pacientes (Bensmail et al., 2010; Sommerfeld et al., 2004) acomete principalmente a musculatura antigravitacional (Dietz e Sinkjaer, 2007; Lima et al., 2008), limitando as atividades da vida diária e interferindo na qualidade de vida dos pacientes (Urban et al., 2010).

Como conceito-chave dentro da reabilitação neurológica (Sorinola et al., 2009), a quantificação exata da espasticidade tem importância tanto diagnóstica quanto terapêutica. Para tanto, dispomos de indicadores quantitativos e qualitativos (Alibiglou et al., 2008; Kumar et al., 2006). Embora as escalas continuem a ser o método mais comum para avaliar a espasticidade (Sorinola et al., 2009), há um considerável potencial em técnicas de instrumentação biomédica que podem proporcionar maior confiabilidade e precisão de quantificação (Johnson, 2002).

A dinamometria isocinética pode ser utilizada com esse objetivo, pois é um instrumento fundamental e útil para analisar a resistência angular ao movimento passivo de modo contínuo numa velocidade superior a $120 \%$, pois gera uma excitabilidade do reflexo de estiramento (Kamper et al., 2001; Kim e Eng, 2003; Lima et al., 2008; Lindberg, 2009; Nuyens et al., 2002). Bem como a Eletromiografia (EMG), método que analisa a atividade elétrica neuromuscular, que é um sinal bioelétrico resultante dos potenciais de ação das fibras musculares, expresso por voltagem em função do tempo captado por eletrodos de superfície em regiões específicas do músculo (Mirbagheri et al., 2007; Nuyens et al., 2002; Sorinola et al., 2009).

A fisioterapia tem um papel primordial no tratamento desses pacientes e dispõe de uma gama de recursos que podem ser utilizados para reduzir o tônus muscular, a fim de promover a recuperação motora e prevenir complicações secundárias (Dietz e Sinkjaer, 2007), dentre os quais se encontram a crioterapia (Akinbo et al., 2007; Allison e Abrahan, 2001; Chesterton et al., 2002; Lee et al., 2002a; Westerlunda et al., 2003) e a EENM (Estimulação Elétrica NeuroMusucular) (Akinbo et al., 2007; Lima et al., 2008).

A crioterapia é um procedimento físico que consiste na transferência de energia térmica através dos tecidos, a fim de diminuir a temperatura de certa região corporal com finalidades terapêuticas (Chesterton et al., 2002; Felice e Santana, 2009). Esta técnica tem sido utilizada recentemente na redução da espasticidade muscular, tanto na prática clínica quanto no meio científico (Allison e Abrahan, 2001; Chesterton et al., 2002; Lee et al., 2002a; Westerlunda et al., 2003). A aplicação terapêutica da crioterapia reduz gradativamente a transmissão dos impulsos nos nervos sensitivos por causa da diminuição da velocidade de condução nervosa. No entanto, após uma aplicação prolongada de compressa fria, a duração dos potenciais de ação dos nervos sensoriais pode aumentar devido a longos períodos refratários (Santuzzi, 2008).

A EENM consiste na aplicação de corrente elétrica (fluxo de elétrons) ao corpo humano com fins terapêuticos (Maffiuletti, 2010; Sheffler e Chae, 2007). Há evidências sobre a efetividade da EENM na redução da espasticidade, quer sobre a musculatura agonista, quer sobre o músculo antagonista ao espástico. Contudo, os mecanismos que fundamentam esses efeitos não são completamente elucidados. Tem sido proposto que a estimulação do antagonista reduz a espasticidade do agonista através da via inibitória recíproca do grupo Ia ou pelas vias polissinápticas mediadas por aferentes dos reflexos flexores. Ao passo que a estimulação do agonista pode levar a uma redução na atividade através da inibição recorrente de seu próprio motoneurônio alfa (Lima et al., 2008). Para tanto, frequência de $50 \mathrm{~Hz}$, duração de pulso de 200-500 $\mu$ s, tempo de sessão de 30 minutos e intensidade variável, entre a que produz apenas o movimento mínimo até a amplitude de movimento completa disponível, são os parâmetros frequentemente utilizados.

Os recursos fisioterápicos supracitados são vantajosos pelos efeitos fisiológicos que proporcionam, 
porém, são insuficientes os trabalhos científicos que forneçam embasamento para o tratamento da espasticidade dos membros superiores, e evidenciem o tempo de duração dos efeitos fisiológicos após aplicação desses recursos. Nesse sentido, o presente trabalho vem contribuir para o estabelecimento de protocolo de aplicação de crioterapia e EENM em indivíduos portadores de hemiparesia espástica, que podem ser utilizados como métodos coadjuvantes no tratamento clínico da espasticidade.

Esse trabalho tem como objetivo analisar e comparar os efeitos da crioterapia e EENM em diferentes instantes (antes, depois, 10, 20 e 30 minutos após as terapias), por meio da resistência ao movimento passivo contínuo e atividade eletromiográfica dos músculos flexores e extensores de cotovelo, em pacientes hemiparéticos espásticos.

\section{Materiais e Métodos}

Trata-se de um Ensaio Clínico Transversal Randomizado, que foi desenvolvido no Laboratório de Biodinâmica da Universidade do Vale do Paraíba (UNIVAP) após aprovação do Comitê de Ética em Pesquisa desta universidade, sob o número de protocolo H 127/CEP 2009.

\section{Casuística}

A amostra foi constituída de 15 indivíduos adultos ( 8 do sexo masculino e 7 do sexo feminino) com diagnóstico médico de Acidente Vascular Encefálico Isquêmico e diagnóstico fisioterapêutico de hemiparesia espástica, com idade média de $56 \pm 16$ anos.

Foram recrutados para a realização do estudo pacientes que estavam em atendimento fisioterapêutico na Clínica de Fisioterapia do Centro de Práticas Supervisionadas (CPS) da UNIVAP, bem como os que aguardavam na lista de espera para a intervenção fisioterapêutica.

Os pacientes foram selecionados de acordo com os seguintes critérios de inclusão:

- Apresentar encaminhamento médico para fisioterapia, que respalda a atuação fisioterapêutica;

- Hemiparesia espástica com no máximo grau 2 de espasticidade nos flexores e extensores de cotovelo segundo a Escala Modificada de Ashworth (Alibiglou et al., 2008; Dietz e Sinkjaer, 2007; Sorinola et al., 2009), pois os graus 3 e 4 referem-se a hipertonia com dificuldade na realização do movimento passivo e rigidez em flexão ou extensão, respectivamente, impossibilitando a realização do teste;
- Amplitude passiva total da articulação do cotovelo ao movimento de flexão e extensão, movimentos exigidos durante o teste com o Dinamômetro Isocinético no modo passivo;

- Deambulação independente com ou sem apoio, para se deslocar até a cadeira do equipamento e posterior posicionamento;

- Cognitivo preservado, sendo capaz de responder ao comando do examinador durante os testes e aplicação dos recursos;

- Mínimo 3 meses de lesão, período em que o aumento do tônus muscular atinge seu máximo (Alibiglou et al., 2008; Dietz e Sinkjaer, 2007; Lieber, 2004; Lindberg, 2009; Sommerfeld et al., 2004).

Como critérios de exclusão foram considerados:

- Infecção ativa e erupções no local de aplicação da EENM, contra-indicação absoluta para sua aplicação;

- Déficit visual e auditivo, que dificultam o fornecimento de informações precisas sobre o nível de estimulação;

- Lesão na área de Wernick, área cerebral responsável pela compreensão da linguagem, tornando-os incapazes de dar feedback durante a intervenção;

- Hiperestesia ou hiperalgesia do grupo muscular a ser avaliado, dificultando a aplicação dos recursos terapêuticos utilizados no estudo;

- Rigidez articular e lesões ósteomioarticulares do cotovelo, que impedem o movimento de flexo-extensão da articulação do cotovelo durante a avaliação dinamométrica;

- Hipertensão arterial não controlada, que está associada a diversas complicações de elevada morbi-mortalidade.

Todos os pacientes foram submetidos à aplicação de crioterapia e EENM. Inicialmente 7 pacientes foram submetidos a crioterapia e 8 pacientes submetidos a aplicação de EENM, divididos de forma aleatória por meio de sorteio. Após uma semana, esses mesmos pacientes foram submetidos à outra terapia que não a submetida inicialmente, totalizando 15 pacientes para cada grupo de intervenção. Todos os indivíduos assinaram espontaneamente o termo de consentimento livre e esclarecido sobre os procedimentos adotados.

\section{Avaliação semiológica, análise biodinâmica e atividade eletromiográfica}

Os indivíduos foram submetidos à avaliação semiológica, que incluiu a quantificação subjetiva da espasticidade por três fisioterapeutas (duplo cego), por meio da Escala de Ashworth Modificada 
(Alibiglou et al., 2008; Dietz e Sinkjaer, 2007; Sorinola et al., 2009).

Em seguida, os indivíduos foram posicionados para realização do teste de resistência ao estiramento passivo produzido pelo Dinamômetro Isocinético Computadorizado (Biodex System ${ }^{\circledR}$ 3) e captação da atividade eletromiográfica (Figura 1). O posicionamento consistiu em sedestação na cadeira do equipamento, com os pés sobre um suporte e estabilizados com cintas de estabilização, uma delas posicionada na coxa do membro inferior sadio e as outras duas cruzadas sobre o tronco. Ao braço de alavanca foi conectado um acessório de punho para posicionar a mão, que foi estabilizada com a utilização de atadura. O cotovelo em $90^{\circ}$ de flexão foi apoiado sobre uma almofada de apoio de membros, e o eixo do dinamômetro foi posicionado de modo que coincidisse com o eixo do movimento de flexo-extensão da articulação do cotovelo. Com o indivíduo posicionado, o dinamômetro foi acionado para que realizasse de modo passivo, uma série de sete movimentos de flexão e extensão da articulação do cotovelo na velocidade angular de $30 \%$ e sete movimentos na velocidade de $150 \%$,

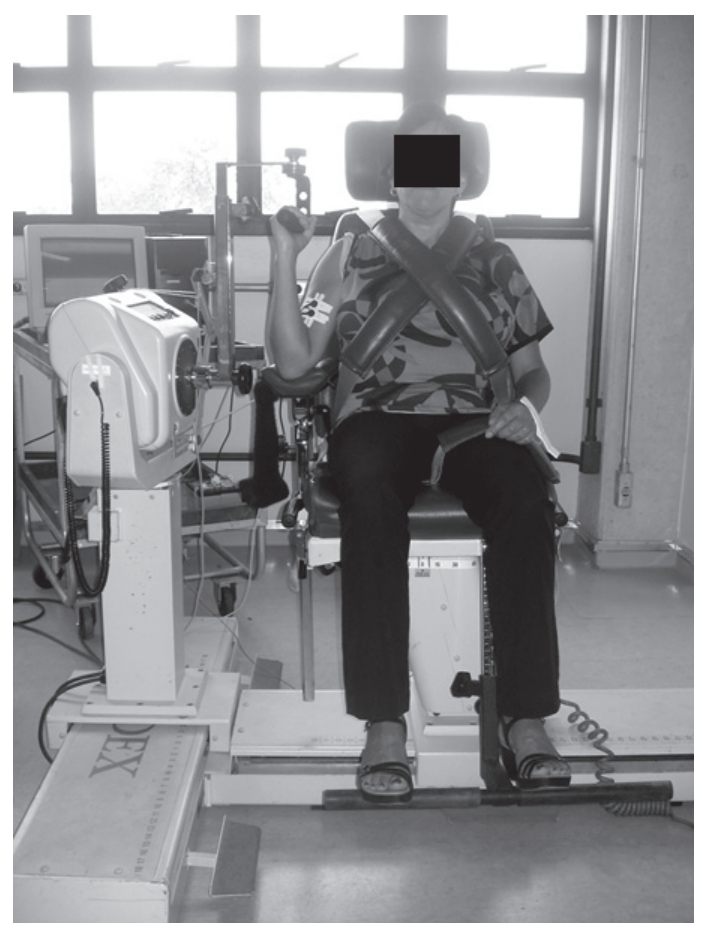

Figura 1. Posicionamento do indivíduo no Dinamômetro Isocinético para avaliação da resistência aos movimentos de flexão e extensão de cotovelo e dos eletrodos para captação da atividade eletromiográfica. Figure 1. Position of the individual on the Isokinetic Dynamometer to evaluate the resistance of elbow flexors and extenders, and location of the electrodes to capture electromyographic activity. com amplitude de movimento de $70^{\circ}$. A velocidade de $150 \%$ s foi utilizada para provocar a excitabilidade do reflexo de estiramento à movimentação passiva, enquanto que a velocidade de $30 \%$ foi utilizada como referência, porque, embora não se observe espasticidade, faz menção a certos fatores que podem exercer influência sobre a resistência ao movimento, como o peso do membro, comprimento do músculo e rigidez articular (Lima et al., 2008).

Os indivíduos foram instruídos a não realizarem qualquer tipo de movimento voluntário ou contração muscular durante o teste, que avaliou a resistência ao movimento de flexão e extensão da articulação do cotovelo. É importante ressaltar que durante o movimento de extensão do cotovelo está sendo avaliada a resistência dos músculos flexores ao movimento, enquanto que no movimento de flexão estão sendo avaliados os músculos extensores de cotovelo.

Simultaneamente à dinamometria foi avaliada a atividade eletromiográfica utilizando-se o eletromiógrafo EMG410C da marca EMG System do Brasil Ltda. Os parâmetros utilizados consistiram em: freqüência de amostragem de $2000 \mathrm{~Hz}$ por canal, unidade de leitura dos canais em $\mathrm{uV}$, filtro passa alta de $20 \mathrm{~Hz}$ e filtro passa baixa de $500 \mathrm{~Hz}$. Fez-se uso de eletrodos de superfície, passivos, $\mathrm{Ag} / \mathrm{AgCl}$ com $10 \mathrm{~mm}$ de diâmetro, posicionados sobre locais específicos dos músculos bíceps braquial e tríceps braquial, de acordo com as recomendações da SENIAM (Surface ElectroMyoGraphy for the Non-Invasive Assessment of Muscles).

Foram utilizados 3 canais do eletromiógrafo, sendo o primeiro para captação da atividade eletromiográfica do músculo bíceps braquial, cujos eletrodos foram colocados $1 / 3$ à partir da fossa cubital na linha entre o acrômio e fossa cubital; o segundo para captação da atividade elétrica do músculo tríceps braquial, sendo os eletrodos posicionados no meio e 2 dedos de largura medial à linha entre a ângulo posterior do acrômio da escápula e o olécrano; e o terceiro canal para conexão de um sincronizador, que foi um dispositivo elaborado pelos alunos do curso de graduação em Engenharia Biomédica da Univap, para obtenção simultânea da atividade eletromiográfica e da resistência ao movimento passivo. A distância entre os eletrodos foi de $20 \mathrm{~mm}$ e o eletrodo de referência foi posicionado na região anterior do punho do membro contralateral ao estudado. Para diminuir possíveis interferências na passagem do estímulo realizou-se anteriormente a colocação dos eletrodos, uma limpeza da epiderme com álcool a 70\%, e tricotomia quando necessário, ao nível dos músculos estudados.

A avaliação com a dinamometria e atividade eletromiográfica foram realizadas antes e após a 
aplicação da crioterapia e EENM, e nos instantes de 10,20 e 30 minutos depois da aplicação do recurso terapêutico.

\section{Protocolo de aplicação}

Os recursos fisioterapêuticos (Figura 2) utilizados foram a EENM e a crioterapia. O gelo foi aplicado na região anterior e medial do braço do hemicorpo acometido (Figura 2a) por meio de sacos plásticos com gelo (Figura 2b), utilizando um pano de limpeza Cross-Hatch ${ }^{\circledR}$ entre a pele do indivíduo e o saco de gelo e uma atadura de crepom para fixá-lo ao braço, por um período de 20 minutos. Para a aplicação da EENM os eletrodos foram fixados no músculo agonista espástico bíceps braquial (Figura 2c) utilizando-se o aparelho Dualpex 961 da marca Quark $^{\circledR}$ (Figura 2d), programado com os seguintes parâmetros: corrente fásica, frequência de estimulação de $50 \mathrm{~Hz}$, largura de pulso de $300 \mu \mathrm{s}$, intensidade máxima tolerada pelo paciente, variando de 20 a $50 \mathrm{~mA}$ e tempo de 15 minutos.

\section{Coleta de dados}

Os dados da resistência ao movimento produzido no movimento passivo das sete repetições na velocidade de $30 \%$ e sete movimentos de flexo-extensão a $150 \%$, foram plotados juntamente com o ângulo de movimento no software EMGworks Analysis, de
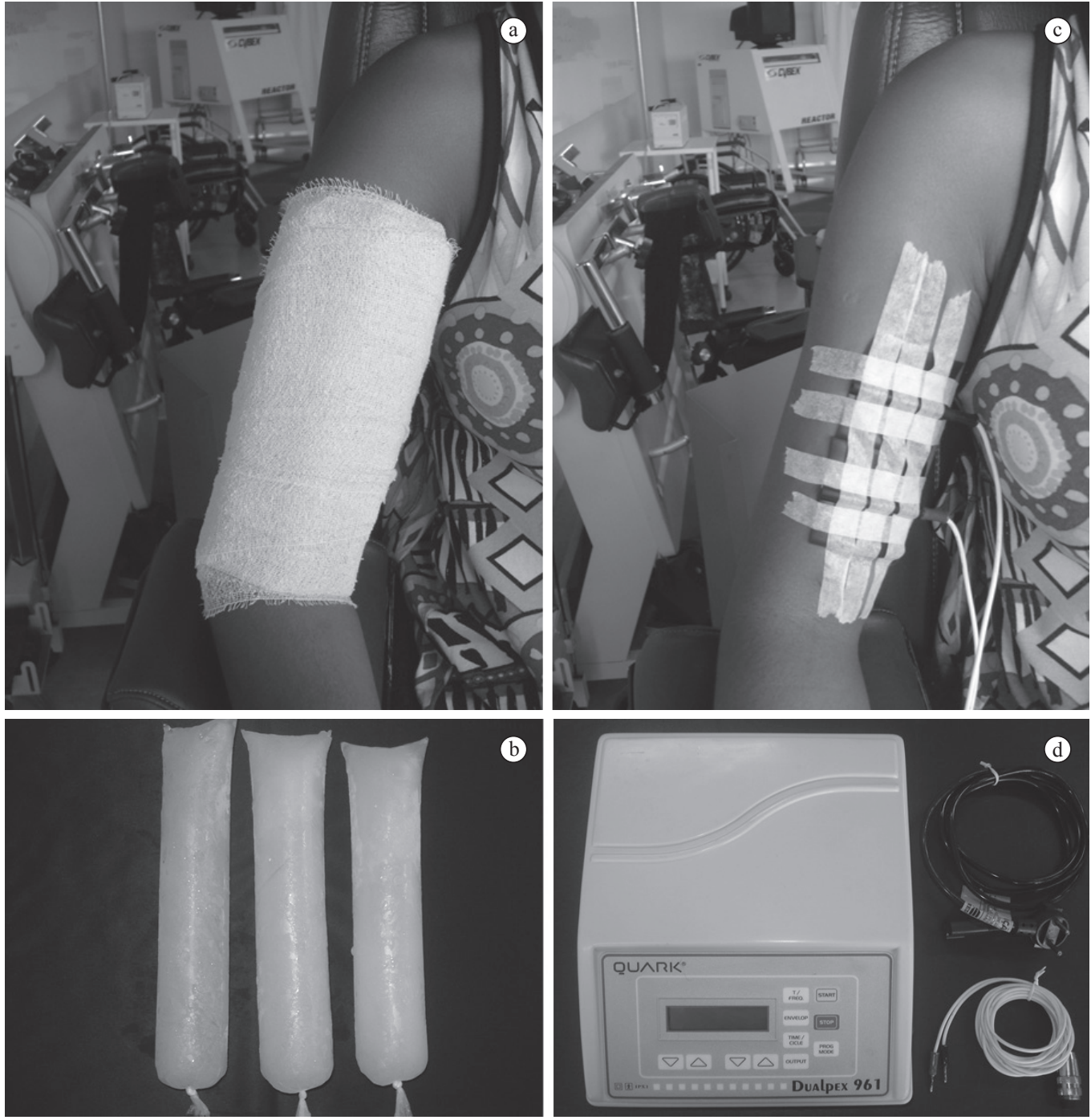

Figura 2. Recursos fisioterapêuticos: a) aplicação de crioterapia na região anterior e medial do braço; b) sacos plásticos com gelo; c) aplicação de EENM no músculo bíceps braquial; e d) Aparelho de EENM, Dualpex 961 da marca Quark ${ }^{\mathbb{B}}$.

Figure 2. Physiotherapy resources: a) application of cryotherapy on the anterior and medial arm; b) plastic bags with ice; c) Application of NMES on the biceps muscle; and d) NMES equipment, Dualpex 961 from Quark ${ }^{\circledR}$. 
modo que, o valor de resistência de cada movimento foi adquirido, sendo excluído o primeiro movimento devido a adaptação do paciente ao teste, realizando-se a média de seis movimentos de flexão e extensão em ambas as velocidades para cada voluntário. Em seguida, os valores médios das seis repetições obtidos na velocidade de $30 \%$, utilizada como referência, foram subtraídos dos valores médios alcançados nas velocidades de $150 \%$ s. Após realizar esse procedimento para cada voluntário, obteve-se a média dos voluntários para os instantes de antes e depois da aplicação, 10, 20 e 30 minutos após o término do emprego do recurso terapêutico.

Os dados referentes à atividade elétrica dos músculos bíceps braquial e tríceps braquial, produzidos durante o movimento de flexão e extensão, foram plotados no software EMGworks Analysis juntamente com o canal do sincronizador, para que fosse possível calcular o valor RMS durante a janela de coleta do dinamômetro, desconsiderado o primeiro movimento.

Os valores quantitativos do sinal eletromiográfico e da resistência ao movimento passivo foram tabulados em planilhas no programa Microsoft Office Excel 2007. Os valores de RMS pós terapia foram normalizados pelos valores obtidos antes das intervenções para posterior análise, realizada por meio do software BioEstat 5.0. Devido a normalização dos valores RMS, os valores dos gráficos e tabelas estão expressos em unidade arbitrária (u.a.).

\section{Análise estatística}

Inicialmente foi realizada a análise descritiva (tamanho da amostra, mínimo e máximo, média aritmética, variância, desvio padrão e erro padrão) a fim de verificar as características específicas e gerais da amostra estudada.

Utilizou-se o teste D'Agostino para analisar a normalidade dos dados visando a escolha do tipo de teste estatístico a ser aplicado (paramétrico ou não paramétrico). A partir desta análise, verificou-se que as variáveis apresentaram distribuição normal, sendo então selecionado o teste paramétrico para análise. Para verificar a existência de diferenças estatísticas entre as médias dos dados nas diferentes fases do experimento (antes, depois, 10, 20 e 30 minutos depois da aplicação) utilizou-se a análise de variância (ANOVA one-way) com nível de significância de $\mathrm{p}<0,05$.

O Teste-t com nível significância de $\mathrm{p}<0,05$ foi utilizado para verificar a existência de diferenças estatísticas entre os recursos utilizados (crioterapia e EENM) para o tratamento da espasticidade, nos instantes antes, depois, 10, 20 e 30 minutos depois.

Posteriormente, calculou-se a porcentagem dos valores de torque e RMS, com base nos valores obtidos antes das terapias, para os instantes depois, 100, 20 e minutos depois do término da aplicação, a fim de direcionar a resposta dos indivíduos espásticos quando submetidos à crioterapia e EENM.

\section{Resultados}

De acordo com a Escala de Ashworth Modificada 40\% dos pacientes apresentaram grau 1 de espasticidade dos flexores de cotovelo, $46,67 \%$ grau $1+$ e 13,33\% grau 2.

Os valores encontrados na análise de variância (ANOVA one-way) e os valores obtidos por meio do Teste $\mathrm{t}$ com nível de significância de $\mathrm{p}<0,05$, não foram estatisticamente significativos.

A seguir são apresentados tabelas e gráficos dos valores de resistência ao movimento passivo e RMS e, descrito a porcentagem de melhora com base nos valores obtidos antes das terapias, para os instantes depois, 10, 20 e 30 minutos após a aplicação, a fim de demonstrar quantitativamente a resposta dos indivíduos espásticos quando submetidos à crioterapia e EENM.

Os valores de resistência ao movimento de flexão e extensão do cotovelo obtidos por meio do Dinamômetro Isocinético no modo passivo, e valores de RMS normalizados para os músculos bíceps braquial e tríceps braquial obtidos por meio da EMG nos instantes de antes, depois, 10, 20 e 30 minutos depois da aplicação de crioterapia e EENM estão apresentados na Tabela 1 e 2, respectivamente.

Os resultados de resistência ao movimento contínuo obtidos por meio do dinamômetro isocinético durante o movimento passivo de extensão e flexão de

Tabela 1. Valores de resistência ao movimento contínuo para extensão e flexão do cotovelo obtidos por meio do Dinamômetro Isocinético no modo passivo $(150 \%$ s), nos instantes de antes, depois, 10, 20 e 30 minutos depois da aplicação de crioterapia e EENM.

Table 1. Values of resistance for the continuous movement of elbow extensors and flexors obtained by the Isokinetic Dynamometer in the passive mode $(150 \%$ s), measured before and after, as well as 10, 20, and 30 minutes following the application of cryotherapy and NMES.

\section{Dinamômetro Isocinético}

\begin{tabular}{|c|c|c|c|c|c|c|c|c|c|c|}
\hline \multirow[t]{2}{*}{ Instantes } & \multicolumn{2}{|c|}{ Antes } & \multicolumn{2}{|c|}{ Depois } & \multicolumn{2}{|c|}{10 minutos depois } & \multicolumn{2}{|c|}{20 minutos depois } & \multicolumn{2}{|c|}{30 minutos depois } \\
\hline & Extensão & Flexão & Extensão & Flexão & Extensão & Flexão & Extensão & Flexão & Extensão & Flexão \\
\hline Crioterapia & $-0,819$ & 2,017 & $-1,249$ & 2,059 & $-1,417$ & 1,845 & $-1,397$ & 1,932 & $-1,186$ & 1,887 \\
\hline EENM & $-0,884$ & 1,982 & $-0,891$ & 1,928 & $-1,082$ & 1,675 & $-1,188$ & 1,912 & $-1,125$ & 1,807 \\
\hline
\end{tabular}


Tabela 2. Valores de RMS (u.a.) para os músculos bíceps braquial e tríceps braquial obtidos por meio da EMG durante o movimento passivo $(150 \%$ s) de extensão e flexão do cotovelo, nos tempos de antes, depois, 10, 20 e 30 minutos depois da aplicação de crioterapia e EENM. Table 2. RMS values (u.a.) for the biceps brachii and triceps brachii obtained by EMG during passive movement $(150 \%$ s) of elbow extensors and flexors, before and after, as well as 10,20, and 30 minutes following the application of cryotherapy and NMES.

\begin{tabular}{|c|c|c|c|c|c|c|c|c|c|c|}
\hline \multicolumn{11}{|c|}{ EMG } \\
\hline Instantes & \multicolumn{2}{|c|}{ Antes } & \multicolumn{2}{|c|}{ Depois } & \multicolumn{2}{|c|}{10 minutos depois } & \multicolumn{2}{|c|}{20 minutos depois } & \multicolumn{2}{|c|}{30 minutos depois } \\
\hline & Bíceps & Tríceps & Bíceps & Tríceps & Bíceps & Tríceps & Bíceps & Tríceps & Bíceps & Tríceps \\
\hline Crioterapia & 1,000 & 1,000 & 1,209 & 0,944 & 1,157 & 1,085 & 1,272 & 1,120 & 1,153 & 1,219 \\
\hline EENM & 1,000 & 1,000 & 1,224 & 0,888 & 1,273 & 0,918 & 1,093 & 0,865 & 1,097 & 0,794 \\
\hline
\end{tabular}

cotovelo quando submetidos à crioterapia e EENM estão demonstrados no Figura 3 e 4, respectivamente.

A crioterapia reduziu em $52,5 \%$ a resistência ao movimento dos flexores de cotovelo após terapia, $73 \% 10$ minutos depois, $70,6 \% 20$ minuto se $44,8 \%$ 30 minutos depois do término da terapia. Em relação à EENM houve uma diminuição da resistência ao movimento de $0,8 \%$ depois da terapia, 22,4\% 10 minutos depois do término da terapia, 34,4\% 20 minutos depois e 27,3\% 30 minutos depois.

Houve um aumento de $2,1 \%$ da resistência ao movimento dos extensores de cotovelo logo após o término da terapia com gelo, porém, ocorreu uma diminuição de $8,5 \% 10$ minutos depois, seguida de $4,2 \%$ e $6,4 \%$ após 20 e 30 minutos depois do término da terapia, respectivamente. A EENM promoveu uma diminuição da resistência ao movimento de $2,7 \%$ depois da terapia, $15,5 \% 10$ minutos depois, $3,5 \% 20$ minutos depois e $8,8 \% 30$ minutos depois do término da terapia.

Estão apresentados em gráficos os valores de RMS normalizados (u.a.), calculados por meio do sinal eletromiográfico, obtidos durante a janela de coleta do dinamômetro na velocidade angular de $150 \%$ dos músculos bíceps braquial (Figura 5) e tríceps braquial (Figura 6).

A crioterapia aumentou em $20,9 \%$ o RMS, sugerindo o recrutamento de unidade motoras dos flexores de cotovelo após terapia, 15,7\% 10 minutos depois, $27,2 \% 20$ minutos e $15,3 \% 30$ minutos depois do término da terapia. A EENM aumentou o RMS em $22,4 \%$ depois da terapia, $27,3 \% 10$ minutos depois do término da terapia, 9,3\% 20 minutos depois e 9,7\% 30 minutos depois.

Houve uma diminuição de 5,6\% do RMS dos extensores de cotovelo depois da terapia com gelo, porém, depois de 10, 20 e 30 minutos após o término da terapia ocorreu um aumento de 8,5\%,12 e 21,9\%, respectivamente. Uma diminuição do recrutamento de unidades motoras foi observada em todos os instantes após a aplicação de EENM, 11,2\% depois da terapia, $8,2 \% 10$ minutos depois, $13,5 \% 20$ minutos depois e $20,6 \% 30$ minutos após o término da terapia.

\section{Discussão}

As disfunções decorrentes do AVE implicam incapacidades funcionais e representam um problema social na vida dos pacientes (Cardoso et al., 2007; Francis et al., 2004). A espasticidade, um dos fatores limitantes funcionais, atinge principalmente a musculatura antigravitacional pós-AVE (Dietz e Sinkjaer, 2007; Lima et al., 2008), afetando consequentemente, o músculo bíceps braquial, que embora exerça função tônica, de suporte do corpo contra a gravidade, é composto predominante de fibras de contração rápida (tipo II) (Dahmane et al., 2005).

Maiores graus de espasticidade pós-AVE são observados nos membros superiores (Urban et al., 2010), porém, sua taxa de recuperação funcional é inferior quando comparada ao treino de marcha independente, sendo necessários métodos eficazes para sua reabilitação (Chan et al., 2009).

O tratamento para minimizar os efeitos da espasticidade continua sendo um dos principais objetivos para os médicos e terapeutas como parte da reabilitação pós-AVE. Dado este foco de investigação, é importante ser capaz de mensurar com precisão a espasticidade, a fim de planejar e monitorar o tratamento (Kumar et al., 2006).

No presente trabalho, a espasticidade dos músculos flexores e extensores de cotovelo pós-AVE foi avaliada com o uso do dinamômetro isocinético associado a eletromiografia de superfície, pois já se sabe que a hipertonia espástica pode ser mensurada pela combinação de torque e atividade eletromiográfica durante movimentos passivos (Aquino et al., 2007; Kamper et al., 2001; Kim e Eng, 2003; Kumar et al., 2006; Mirbagheri et al., 2007; Nuyens et al., 2002).

Semelhantes ao método de avaliação utilizados nessa pesquisa, Lee et al. (2002b) analisaram o torque dos músculos flexores de cotovelo em pacientes hemiparéticos, parkinsonianos e indivíduos normais nas velocidades de $40,80,120$ e $160 \%$ s, numa amplitude de movimento de $75^{\circ}$ de flexão e extensão de cotovelo. Simultaneamente ao torque, coletaram a atividade eletromiográfica dos músculos bíceps 


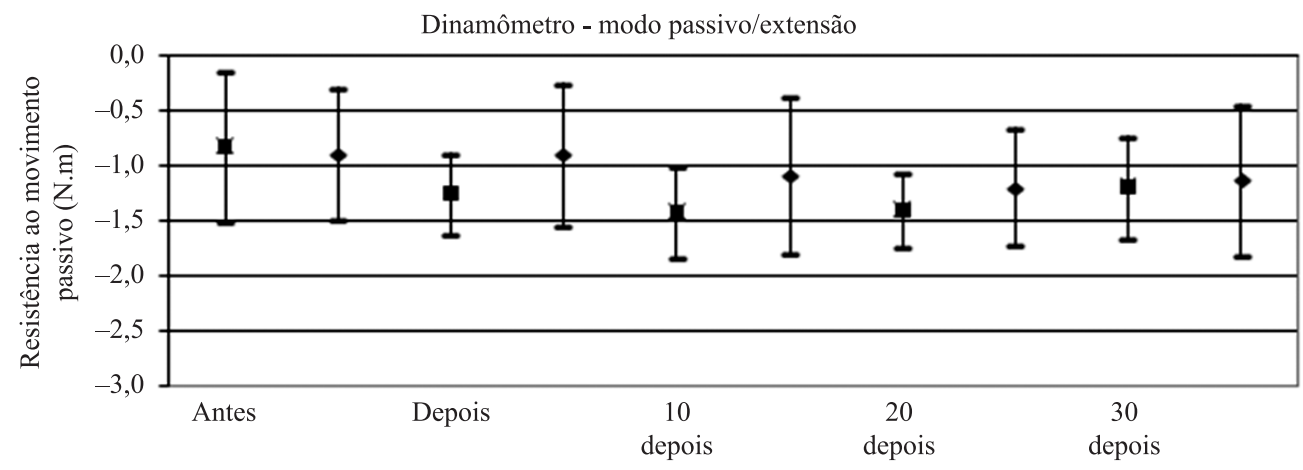

Tempo (minutos)

EENM Crioterapia

Figura 3. Resistência ao movimento passivo de extensão do cotovelo antes, após e 10, 20 e 30 minutos depois da aplicação de crioterapia e EENM.

Figure 3. Resistance to passive movement of elbow extensors before and after, as well as 10, 20, and 30 minutes following the application of cryotherapy and electrical stimulation.

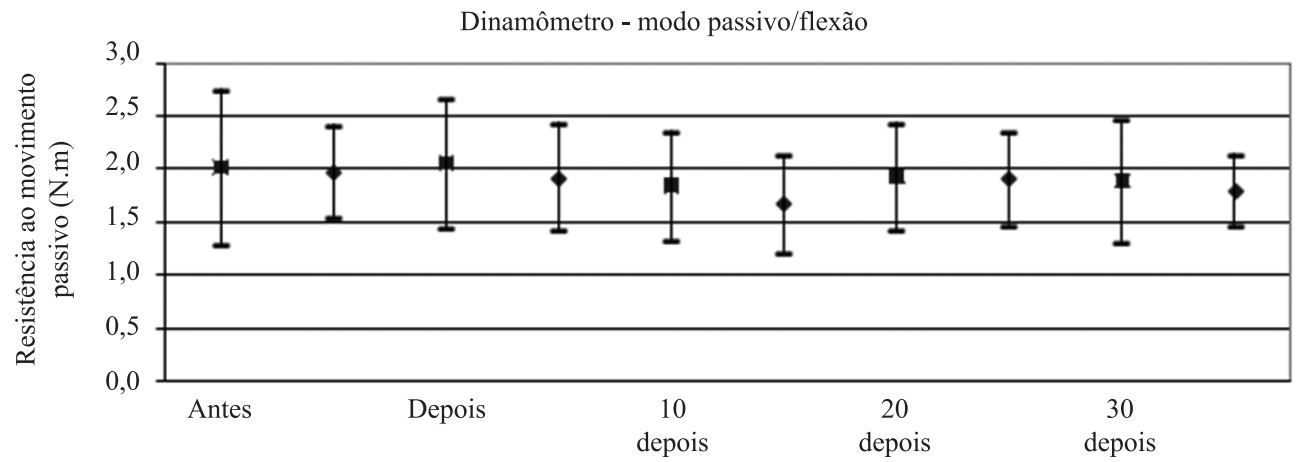

Tempo (minutos)

- EENM $\mathbf{a}$ Crioterapia

Figura 4. Resistência ao movimento passivo de flexão de cotovelo antes, após e nos instantes de 10, 20 e 30 minutos seguidos da aplicação de crioterapia e EENM.

Figure 4. Resistance to passive movement of elbow flexors before and after, as well as 10, 20, and 30 minutes following the application of cryotherapy and electrical stimulation.

e tríceps braquial. Porém, o objetivo do trabalho era determinar o padrão de resistência durante o alongamento passivo dos flexores de cotovelo nas populações estudadas, sendo possível observar que a resistência é mais pronunciada nos indivíduos com alterações do tônus muscular, e que o aumento do tônus é progressivo na espasticidade, ao passo que na rigidez ocorre um aumento constante.

Métodos fisioterapêuticos devem ser aplicados sobre o membro espástico para reduzir o tônus muscular, a fim de promover a recuperação motora do membro superior comprometido e prevenir complicações secundárias (Dietz e Sinkjaer, 2007). Embora, a escolha do método seja difícil na prática clínica (Kumar et al., 2006; Romero et al., 2008) diversos recursos estão disponíveis, dentre os quais se encontram a crioterapia e a EENM (Akinbo et al., 2007).

Nossos resultados, embora, não estatisticamente significativos (ANOVA e Teste-t) nos permitem demonstrar a tendência de resposta dos indivíduos espásticos quando submetidos aos dois recursos terapêuticos propostos, nos diferentes instantes investigados, por meio da porcentagem dos valores de resistência ao movimento passivo e valores de RMS do sinal eletromiográfico. 


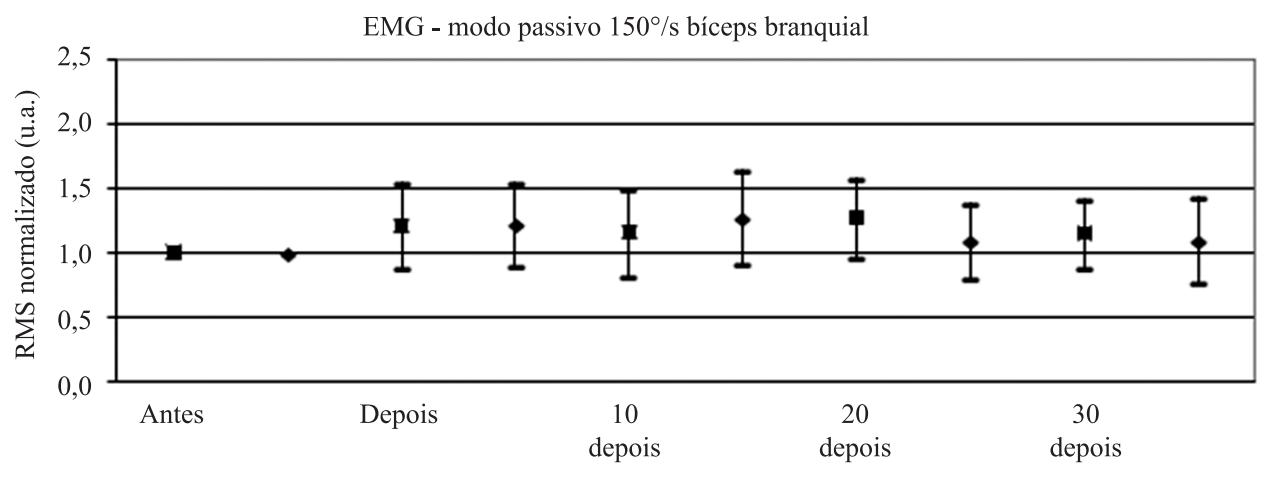

Tempo (minutos)

EENM - Crioterapia

Figura 5. RMS do músculo bíceps braquial durante a avaliação da resistência ao movimento de flexão e extensão do cotovelo na velocidade angular de $150 \%$ antes, após e nos instantes de 10, 20 e 30 minutos seguidos do término da aplicação de crioterapia e EENM.

Figure 5. RMS of the brachial biceps during the test of resistance to movement of the elbow flexors and extensors in angular velocity of $150 \%$ before and after, as well as 10,20, and 30 minutes following application of cryotherapy and NMES.

EMG - modo passivo $150 \%$ s tríceps branquial

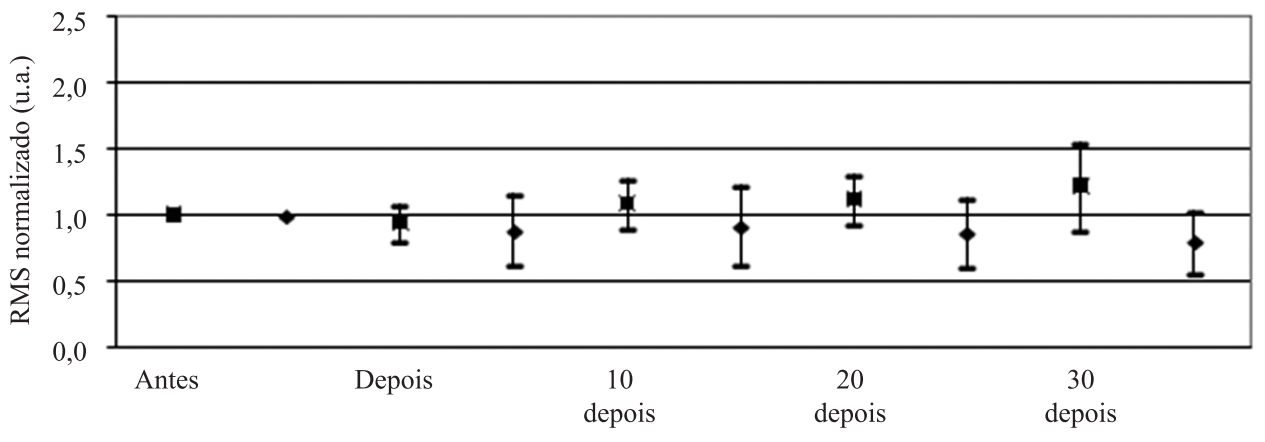

Tempo (minutos)

$\bullet$ EENM $\mathbf{a}$ Crioterapia

Figura 6. RMS do músculo tríceps braquial obtido durante a avaliação da resistência ao movimento de flexão e extensão do cotovelo na velocidade angular de $150 \%$ s antes, após e nos instantes de 10, 20 e 30 minutos seguidos do término da aplicação de crioterapia e EENM. Figure 6. RMS of the brachial triceps during test for resistance to movement of the elbow flexors and extensors in angular velocity of $150 \%$ before and after, as well as 10,20, and 30 minutes following application of cryotherapy and NMES.

A crioterapia reduziu percentualmente a resistência ao movimento dos músculos espásticos flexores de cotovelo, e seu efeito perdurou por até 30 minutos depois do término da terapia, tendo seu efeito máximo aos 10 minutos depois do término da terapia. Segundo Felice e Santana (2009) os efeitos da crioterapia na redução da espasticidade podem perdurar por um período que varia de 30 minutos a 2 horas. Esses resultados também corroboram com os apresentados por Lee et al. (2002a), quando afirmaram em seu estudo em coelhos com lesão na medula espinal que a crioterapia foi efetiva na redução imediata da espasticidade e 30 minutos depois da aplicação.
A resistência ao movimento passivo do músculo tríceps braquial aumentou logo após a terapia, porém, nos 30 minutos seguintes apresentou uma diminuição. Os dados da EMG em $150 \%$ s complementam esses achados, pois revelam um aumento do RMS 10,20 e 30 minutos pós-crioterapia, que sugere maior número de unidades motoras ativadas (De Luca et al., 2006). Essa diminuição da resistência ao movimento passivo e o aumento da atividade eletromiográfica nos permite sugerir que houve uma diminuição da hipertonia espástica pós-tratamento e melhora da função muscular do antagonista ao espástico, promovendo o sinergismo muscular. 
Na velocidade de $30 \%$ s observou-se diminuição do RMS dos extensores de cotovelo. Esse fato pode ser explicado pelo estiramento constante do tríceps braquial devido ao padrão flexor do bíceps braquial, que gera uma fraqueza muscular e consequente diminuição da atividade muscular. Pois na velocidade de $30 \%$ observa-se uma resposta mecânica do músculo, enquanto que na velocidade de $150 \%$ s há estiramento do fuso muscular.

Santos et al. (2004) observaram diminuição da espasticidade no músculo masseter em crianças com paralisia cerebral após a crioterapia, devido a uma redução na velocidade de condução dos nervos sensoriais, ocorrendo também uma queda na transmissão sináptica propiciada pela baixa temperatura.

A EENM também reduziu percentualmente a resistência ao movimento do músculo bíceps braquial, que pode ser observada pela diminuição da resistência a movimentação passiva e aumento dos valores de RMS obtidos na velocidade angular de $150 \% \mathrm{~s} \mathrm{em}$ todos os instantes analisados. Porém, a diminuição da resistência foi mais evidente nos instantes de 10,20 e 30 minutos após o término da terapia, sugerindo um efeito tardio na espasticidade.

A EENM além de promover a redução da espasticidade propiciou uma redução da resistência a movimentação passiva do músculo antagonista ao espástico, porém, os valores de RMS apresentaram diminuição. Segundo Lamontagne et al. (1998) uma redução na resistência durante movimentos passivos repetidos sem alterações simultâneas na atividade eletromiográfica podem ser atribuídas às características tixotrópicas dos tecidos esticados, que refere-se à propriedade de certos sistemas de tornar-se menos viscoso quando abalado e, em seguida, retornar a viscosidade inicial.

A redução da hipertonia espástica após aplicação de EENM já pôde ser observada em estudos anteriores. Van der Salm et al. (2006) observaram redução da espasticidade do músculo tríceps sural em pacientes com lesão medular completa após a utilização de EENM. Lima et al. (2008) relataram efeitos satisfatórios dos exercícios dinâmicos e da EENM na redução da espasticidade dos músculos flexores e extensores do joelho, por meio dos dados obtidos no modo passivo $(120 \%$ s) do dinamômetro isocinético.

Akinbo et al. (2007) avaliaram os efeitos a curto prazo da crioterapia e EENM em crianças com paralisia cerebral, e observaram uma redução significativa da espasticidade de flexores de punho e dedos quando tratados com crioterapia, porém, para pacientes tratados com EENM, a redução da espasticidade não foi significativa.
Ao contrário dos resultados observados por Akinbo et al. (2007), Martins (2009) afirma que a TENS (Estimulação Elétrica Nervosa Transcutânea) pode ser um importante recurso para redução imediata da espasticidade e que a crioterapia aumenta o estado de hiperreflexia dos pacientes espásticos. Embora a corrente elétrica terapêutica utilizada por Martins (2009) não seja compatível com a utilizada por Akinbo et al. (2007), a frequência de estimulação é o único parâmetro que as diferencia.

\section{Conclusões}

Diante dos resultados da presente pesquisa concluímos que:

- Apesar de não significativos, os resultados sugerem que os recursos terapêuticos utilizados promovem redução da resistência à movimentação passiva dos flexores e extensores de cotovelo. A crioterapia apresentou resultados mais satisfatórios e teve seu efeito máximo na redução da espasticidade do músculo bíceps braquial 10 minutos depois do término da terapia $(59,8 \%)$, ao passo que a EENM apresentou melhores resultados 20 e 30 minutos após o término da terapia $(30,4$ e $24,1 \%$, respectivamente), sugerindo um efeito agudo da crioterapia e um efeito tardio da EENM.

- A redução da resistência à movimentação passiva dos músculos flexores e extensores de cotovelo após aplicação da crioterapia pode ser atribuída à redução da transmissão sináptica devido à diminuição da velocidade de condução nervosa, enquanto que a efetividade da EENM se deve, provavelmente, a uma redução na atividade por meio da inibição recorrente de seu próprio motoneurônio alfa (Lima et al., 2008).

- Os protocolos de aplicação de crioterapia e EENM utilizados no presente estudo podem ser utilizados como métodos coadjuvantes no tratamento clínico da espasticidade.

- São necessários estudos com uma amostra maior, para esclarecer melhor os efeitos dessas terapias.

\section{Agradecimentos}

Os autores agradecem a CAPES pelo apoio financeiro.

\section{Referências}

Akinbo SRA, Tella BA, Otunla AB, Temiye EO. Comparison of the effect of neuromuscular electrical stimulation and cryotherapy on spasticity and hand function 
in patients with spastic cerebral palsy. Nigerian Medical Practitioner. 2007; 51(6):128-32. http://dx.doi.org/10.4314/ nmp.v51i6.28858

Alibiglou L, Rymer WZ, Harvey RL, Mirbagheri MM. The relation between Ashworth scores and neuromechanical measurements of spasticity following stroke. Journal of NeuroEngineering and Rehabilitation. 2008; 15:5-18.

Allison SC, Abraham LD. Sensitivity of qualitative and quantitative spasticity measures to clinical treatment with cryotherapy. International Journal of Rehabilitation Research. 2001; 24:15-24. PMid:11302460. http://dx.doi. org/10.1097/00004356-200103000-00003

Aquino CF, Vaz DV, Brício RS, Silva PLP, Ocarino JM, Fonseca ST. A Utilização da dinamometria isocinética nas ciências do esporte e reabilitação. Revista Brasileira de Ciência e Movimento. 2007; 15(1):93-100

Bensmail D, Robertson JVG, Fermanian C, Roby-Bramiet A. Botulinum toxin to treat upper-limb Spasticity in hemiparetic patients: analysis of function and kinematics of reaching movements. Neurorehabilitation and Neural Repair. 2010; 24(3):273-81. PMid:20067949. http://dx.doi. org/10.1177/1545968309347682

Cardoso E, Pedreira G, Prazeres A, Ribeiro N, Melo A. Does botulinum toxin improve the function of the patient with spasticity after stroke? Arquivos de Neuro-Psiquiatria. 2007; 65(3a):592-5. PMid:17876396. http://dx.doi.org/10.1590/S0004-282X2007000400008

Chan MK-1, Tong RK-Y, Chung KY-K. Bilateral upper limb training with functional electric stimulation in patients with chronic stroke. Neurorehabilitation and Neural Repair. 2009; 23(4):357-65. PMid:19074684.

Chesterton LS, Foster NE, Ross L. Skin temperature response to cryotherapy. Archives of Physical Medicine and Rehabilitation. 2002; 83:543-9. PMid:11932859. http:// dx.doi.org/10.1053/apmr.2002.30926

Dahmane R, Djordjevič S, Šimunič B, Valenčič V. Spatial fiber type distribution in normal human muscle - Histochemical and tensiomyographical evaluation. Journal of Biomechanics. 2005; 38:2451-9. PMid:16214493. http:// dx.doi.org/10.1016/j.jbiomech.2004.10.020

De Luca CJ, Adam A, Wotiz R, Gilmore LD, Nawab $\mathrm{SH}$. Decomposition of surface EMG signals. Journal of neurophysiology. 2006; 96:1646-57. PMid:16899649. http:// dx.doi.org/10.1152/jn.00009.2006

Dietz V, Sinkjaer T. Spastic movement disorder: impaired reflex function and altered muscle mechanics: Review. Lancet Neurology. 2007; 6:725-33. PMid:17638613.

Felice TD, Santana LR. Recursos fisioterapêuticos (crioterapia e termoterapia) na espasticidade: revisão de literatura. Revista Neurociências. 2009; 17(1):57-62.

Francis HP, Wade DT, Turner-Stokes L, Kingswell RS, Dott CS, Coxon EA. Does reducing spasticity translate into functional benefit? An exploratory meta-analysis. Journal of Neurology, Neurosurgery and Psychiatry. 2004; 75:1547-51. PMid:50411. http://dx.doi.org/10.1136/jnnp.2003.025551
Johnson GR. Outcome measures of spasticity. European Journal of Neurology. 2002; 9(S1):10-6.

Kamper DG, Schmit BD, Rymer WZ. Effect of muscle biomechanics on the quantification of spasticity. Annals of Biomedical Engineering. 2001; 29:1122-34. PMid:11853265. http://dx.doi.org/10.1114/1.1424918

Kim CM, Eng JJ. The relationship of lower-extremity muscle torque to locomotor performance in people with stroke. Physical Therapy. 2003; 83(1):49-57. PMid:12495412.

Kumar RTS, Pandyan AD, Sharma AK. Biomechanical measurement of post-stroke spasticity. Age and Ageing. 2006; 35:371-5. PMid:16675479. http://dx.doi. org/10.1093/ageing/afj084

Lamontagne A, Malouin F, Richards CL, Dumas F. Evaluation of reflex- and nonreflex-induced muscle resistance to stretch in adults with spinal cord injury using hand-held and isokinetic dynamometry. Physical Therapy. 1998; 78(9):964-78. PMid:9736894.

Lee S-U, Bang MS, Han TR. Effect of cold air therapy in relieving spasticity: applied to spinalized rabbits. Spinal Cord. 2002a; 40:167-73. PMid:11965554. http://dx.doi. org/10.1038/sj.sc.3101279

Lee H-M, Huang Y-Z, Chen J-JJ, Hwang I-S. Quantitative analysis of the velocity related pathophysiology of spasticity and rigidity in the elbow flexors. Journal of Neurology, Neurosurgery and Psychiatry. 2002b; 72:621-9. PMid:50411. http://dx.doi.org/10.1136/jnnp.72.5.621

Lieber RL, Steinman S, Barash I, Chambers H. Structural and functional changes in spastic skeletal muscle. Muscle \& Nerve. 2004; 29(5):615-27. http://dx.doi.org/10.1002/ mus. 20059

Lima MO, Lima FPS, Freitas STT, Ribeiro SR, Tortoza C, Lucareli JG, Lucareli PR, Pacheco MT, Martins RAL. Efecto de la estimulación eléctrica neuromuscular y de los ejercicios isotónicos en los músculos flexores y extensores de la rodilla en pacientes hemipléjicos. Revista de Neurología. 2008; 46(3):135-8. PMid:18297618.

Lindberg PG, Gäverth J, Fagergren A, Fransson P, Forssberg H, Borg J. Cortical activity in relation to velocity dependent movement resistance in the flexor muscles of the hand after stroke. Neurorehabilitation and Neural Repair. 2009; 23(8):800-10. PMid:19470806. http://dx.doi. org/10.1177/1545968309332735

Maffiuletti NA. Physiological and methodological considerations for the use of neuromuscular electrical stimulation. European Journal of Applied Physiology. 2010; 110:223-34. PMid:20473619. http:// dx.doi.org/10.1007/s00421-010-1502-y

Martins FL. Efeitos imediatos da eletroestimulação nervosa transcutânea e crioterapia na espasticidade e na atividade eletromiográfica de sujeitos hemiparéticos [dissertação]. Natal: Universidade Federal do Rio Grande do Norte; 2009. 82 p.

Mirbagheri MM, Settle K, Harvey R, Rymer WZ. Neuromuscular abnormalities associated with spasticity of upper extremity muscles in hemiparetic stroke. Journal of Neurophysiology. 2007; 98:629-37. PMid:17537910. http://dx.doi.org/10.1152/jn.00049.2007 
Nuyens GE, De Weerdt WJ, Spaepen Junior AJ, Kiekens C, Feys HM. Reduction of spastic hypertonia during repeated passive knee movements in stroke patients. Archives of Physical Medicine and Rehabilitation. 2002; 83:930-5. PMid:12098152. http://dx.doi.org/10.1053/apmr.2002.33233

Romero JR, Morris J, Pikula A. Review: Stroke prevention: modifying risk factors. Therapeutic Advances in Cardiovascular Disease. 2008; 2(4):287-303. PMid:19124428 PMCid:2729177. http://dx.doi. org/10.1177/1753944708093847

Santos MTBR, Oliveira LM. Use of cryotherapy to enhance mouth opening in patients with cerebral palsy. Special Care in Dentistry. 2004; 24(4):232-4. PMid:15462553. http:// dx.doi.org/10.1111/j.1754-4505.2004.tb01698.x

Santuzzi CH, Gonçalves WLS, Rocha SS, Castro MEC, Gouvea SA, Abreu GR. Effects of cryotherapy, transcutaneous electrical stimulation and their combination on femoral nerve electrical activity in rats. Revista Brasileira de Fisioterapia. 2008; 12(6)441-6. http://dx.doi.org/10.1590/ S1413-35552008005000007

Sheffler LR, Chae J. Neuromuscular electrical stimulation in neurorehabilitation. Muscle and Nerve. 2007; 35:562-90. http://dx.doi.org/10.1002/mus.20758

Sommerfeld DK, Eek EU-B, Svensson A-K, Holmqvist LW, Arbin MH. Spasticity after stroke: Its occurrence and association with motor impairments and activity limitations. stroke. 2004; 35:134-40. PMid:14684785. http://dx.doi. org/10.1161/01.STR.0000105386.05173.5E

Sorinola IO, White CM, Rushton DN, Newham DJ. Electromyographic response to manual passive stretch of the hemiplegic wrist: Accuracy, reliability, and correlation with clinical spasticity assessment and function. Neurorehabilitation and Neural Repair. 2009; 23(3):287-94. PMid:19050174. http://dx.doi.org/10.1177/1545968308321778

Urban PP, Wolf T, Uebele M, Marx JJ, Vogt T, Stoeter P, Bauermann T, Weibrich C, Vucurevic GD, Schneider A, Wissel J. Occurence and clinical predictors of spasticity after ischemic stroke. Stroke. 2010; 41:2016-20. PMid:20705930. http://dx.doi.org/10.1161/STROKEAHA.110.581991

Van Der Salm A, Veltink PH, IJzerman MJ, GroothuisOudshoorn KC, Nene AV, Hermens HJ. Comparison of electric stimulation methods for reduction of triceps surae spasticity in spinal cord injury. Archives of Physical Medicine and Rehabilitation. 2006; 87:222-8. PMid:16442976. http:// dx.doi.org/10.1016/j.apmr.2005.09.024

Westerlunda T, Oksab J, Smolanderc J, Mikkelsson M. Thermal responses during and after whole-body cryotherapy $\left(-110^{\circ} \mathrm{C}\right)$. Journal of Thermal Biology. 2003; 28:601-8.

World Health Organization - WHO. Neurological disorders: public health challenges. Geneva: WHO; 2006. p. 163-75.

\footnotetext{
Autores

Débora Daisy da Silva*, Ana Carolina Lacerda Borges, Mário Oliveira Lima, Fernanda Pupio Silva Lima, Sérgio Takeshi Tatsukawa De Freitas, Daniel Vilela Nogueira, Alderico Rodrigues de Paula Junior, José Carlos Cogo

Instituto de Pesquisa e Desenvolvimento - IP\&D, Universidade do Vale do Paraíba - UNIVAP, Av. Shishima Hifumi, 2911, CEP 12244-000, Urbanova, São José dos Campos, SP, Brasil

Paulo Roberto Garcia Lucareli

Laboratório de Análise do Movimento, Hospital Israelita Albert Einstein.

Programa de Mestrado em Ciências da Reabilitação, Universidade Nove de Julho - UNINOVE,

Av. Francisco Matarazzo, 612, $1^{\circ}$ andar, CEP 05001-100, Água Branca, São Paulo, SP, Brasil
} 